\title{
Optimal Precoding for a QoS Optimization Problem in 2-user MISO-NOMA Downlink
}

\author{
Zhiyong Chen, Zhiguo Ding, Peng Xu, Xuchu Dai
}

\begin{abstract}
In this letter, based on the NOMA concept, a qualityof-service (QoS) optimization problem for 2-user multiple-inputsingle-output (MISO) broadcast systems is considered, given a pair of target interference levels. The minimal power and the optimal precoding vectors are obtained by considering its Lagrange dual problem and via Newton's iterative algorithm, respectively. Moreover, closed-form expressions of the minimal transmission power for some special cases are also derived. One of these cases is termed as quasi-degraded, which is the key point and will be discussed in detail in this letter. Our analysis further figures out that the proposed NOMA scheme can approach nearly the same performance as optimal dirty paper coding (DPC), as verified by computer simulations.
\end{abstract}

Index Terms-5G, non-orthogonal multiple access, multipleinput-single-output, quality-of-service, precoding.

\section{INTRODUCTION}

$\mathbf{S}$ EEKING efficient multiple access technologies in wireless communication systems has been always an important topic, since it is key to meet with the demand of exponentially increasing data traffic [1]. Recently, NOMA has been proposed for downlink scenarios in 3rd generation partnership project long-term evolution (3GPP-LTE) systems [2]. Moreover, NOMA has also been recognized as a promising candidate for $5 \mathrm{G}$ wireless systems due to its superior spectral efficiency compared to traditional orthogonal multiple access (OMA) [3]-[5]. In consideration of co-channel interference and implementation complexity, a hybrid multiple access system was proposed in [4], in which the users are divided into multiple groups. NOMA is implemented within each group and OMA is carried out between different groups. Consequently, in this paper, we will focus on 2-user MISO-NOMA broadcast systems.

It is well known that the capacity region of degraded broadcast channels can be achieved by utilizing superposition coding (SC) in combination with successive interference cancellation (SIC), i.e, NOMA [6]. Unfortunately, multiple-input-multipleoutput (MIMO) broadcast channels are not generally degraded, of which the capacity region can only be attained by using DPC [7], [8]. However, DPC is difficult to implement in practice due to its nonlinearity and prohibitive complexity. Thus, NOMA schemes with a linear superposition code for

Manuscript received XXX XX, 2016. The associate editor coordinating the review of this letter and approving it for publication was XXX.

This work was supported in part by the National Natural Science Foundation of China (No. 61471334) and the National High Technology Research and Development Program of China (863 Program) with Grant No. 2012AA01A502. The work of Z. Ding was supported by the Royal Society International Exchange Scheme and the UK EPSRC under grant number EP/N005597/1.

Z. Chen, P. Xu and X. Dai are with the Key Lab of Wireless-Optical Commun., Chinese Acad. of Sciences, Sch. of Info. Science \& Tech., Univ. Science \& Tech. China, Hefei, Anhui, 230026, P. R. China. Z Ding is with the School of Computing and Commun., Lancaster Univ., LA1 4YW, UK. (email: zhiyong@mail.ustc.edu.cn, z.ding@lancaster.ac.uk, \{mxp484,daixc\}@ustc.edu.cn).

Digital Object Identifier XXXX/XXXX non-degraded broadcast channels attracts strong research interests. For example, [9] firstly studied the ergodic capacity maximization problem for MIMO-NOMA systems with statistical channel state information at the transmitter. In [10], the downlink sum rate maximization problem for MISO-NOMA systems was studied. The application of simultaneous wireless information and power transfer (SWIPT) to NOMA networks was investigated in [11]. In [12], a minimum power beamforming problem was formulated for MISO-NOMA broadcast systems when the target rates are given. However, the problem was directly optimized via an iterative algorithm based on a nonlinear Gauss-Seidel (GS) algorithm and the closed-form solution was obtained only when the direction of beamforming vectors were given. Moreover, the global optimality was not guaranteed.

This letter considers the precoding problem in 2-user MISONOMA downlink scenarios, where there are predefined interference levels (lower bounds) at the users. The contributions of this paper are two-fold, as listed in the following:

1): In contrast to the existing works, by categorizing the users' channel conditions, closed-form expressions of the minimal transmission power for quasi-degraded channels are obtained. The closed-form solutions not only reduce the computational complexity for the design of optimal precoding, but also yield insightful understandings of MISO-NOMA downlink transmission.

2): Unlike the existing works in [9], [10] and [12], the gap between the optimal performance achieved by DPC and the one achieved by the proposed precoding scheme is clearly identified. In particular, we focus on a heterogenous case, and consider that users' channel conditions are distinctive, an assumption valid for many practical communication scenarios. The developed analytical results demonstrate that the obtained solution for the formulated problem achieves exactly the same performance as DPC, i.e., the performance gap between the proposed scheme and DPC is zero for such heterogenous scenarios. It is also worthy pointing out that the complexity of the proposed scheme is much smaller than DPC, due to the use of superposition coding and SIC.

\section{Problem Formulation}

Consider a 2-user MISO broadcast system with flat fading channels, in which the base station (BS) is equipped with $N$ transmit antennas and each receiver is equipped with single antenna. The system model can be characterized as

$$
y_{i}=\mathbf{h}_{i}^{H} \mathbf{x}+n_{i}, \quad i=1,2,
$$

where $\mathbf{h}_{i} \sim \mathcal{C N}\left(0, \sigma_{i}^{2} \mathbf{I}_{N}\right)$ is the channel coefficient, $\mathbf{h}_{i}^{H}$ denotes the Hermitian transpose of $\mathbf{h}_{i}$, and $n_{i} \sim \mathcal{C N}(0,1)$ is the additive Gaussian noise of user $i$. The BS is intended to convey $s_{i}$ to user $i$ at each time sample. In downlink NOMA, $s_{1}$ and $s_{2}$ are superposed as $\mathbf{x}=\mathbf{w}_{1} s_{1}+\mathbf{w}_{2} s_{2}$, where the 
power of $s_{i}$ is normalized, i.e., $\mathcal{E}\left\{\left\|s_{i}\right\|^{2}\right\}=1$, and $\mathbf{w}_{i}$ is the precoding vector. Given a decoding order $\left(s_{2}, s_{1}\right)^{1}$, by performing SIC at the receiver side, the individual achievable rate of each user can be expressed as [3]

$$
\left\{\begin{array}{l}
R_{1}=\ln \left(1+\mathbf{h}_{1}^{H} \mathbf{w}_{1} \mathbf{w}_{1}^{H} \mathbf{h}_{1}\right), \\
R_{2}=\min \left\{\ln \left(1+\mathrm{SINR}_{2,1}\right), \ln \left(1+\mathrm{SINR}_{2,2}\right)\right\},
\end{array}\right.
$$

where

$\mathrm{SINR}_{2,1}=\frac{\mathbf{h}_{1}^{H} \mathbf{w}_{2} \mathbf{w}_{2}^{H} \mathbf{h}_{1}}{1+\mathbf{h}_{1}^{H} \mathbf{w}_{1} \mathbf{w}_{1}^{H} \mathbf{h}_{1}}, \mathrm{SINR}_{2,2}=\frac{\mathbf{h}_{2}^{H} \mathbf{w}_{2} \mathbf{w}_{2}^{H} \mathbf{h}_{2}}{1+\mathbf{h}_{2}^{H} \mathbf{w}_{1} \mathbf{w}_{1}^{H} \mathbf{h}_{2}}$.

We, herein, consider a quality-of-service (QoS) optimization problem given a pair of target interference levels $r_{1}, r_{2}$. Since the power of $s_{i}$ is normalized, the total power consumption to support reliable transmission is $\left\|\mathbf{w}_{1}\right\|^{2}+\left\|\mathbf{w}_{2}\right\|^{2}$. Consequently, the optimal precoding problem to minimize the total transmission power can be formulated as

$$
\begin{array}{ll}
\min & \left\|\mathbf{w}_{1}\right\|^{2}+\left\|\mathbf{w}_{2}\right\|^{2} \\
\text { s.t. } & R_{1} \geq \ln \left(1+r_{1}\right), R_{2} \geq \ln \left(1+r_{2}\right) .
\end{array}
$$

For clarity purpose, we consider the equivalent optimization problem in (3), which can be formulated as

$$
\begin{array}{ll} 
& p^{*}=\min \quad\left\|\mathbf{w}_{1}\right\|^{2}+\left\|\mathbf{w}_{2}\right\|^{2} \\
\text { s.t. } & \mathbf{h}_{1}^{H} \mathbf{w}_{1} \mathbf{w}_{1}^{H} \mathbf{h}_{1} \geq r_{1} \\
& \mathbf{h}_{1}^{H} \mathbf{w}_{2} \mathbf{w}_{2}^{H} \mathbf{h}_{1} \geq r_{2}+r_{2} \mathbf{h}_{1}^{H} \mathbf{w}_{1} \mathbf{w}_{1}^{H} \mathbf{h}_{1} \\
& \mathbf{h}_{2}^{H} \mathbf{w}_{2} \mathbf{w}_{2}^{H} \mathbf{h}_{2} \geq r_{2}+r_{2} \mathbf{h}_{2}^{H} \mathbf{w}_{1} \mathbf{w}_{1}^{H} \mathbf{h}_{2},
\end{array}
$$

where $p^{*}$ denotes the minimal required power to support the target QoS transmission.

\section{Proposed Iterative Algorithm}

The Lagrangian function for (4) is [13]

$$
\begin{aligned}
& \mathcal{L}\left(\mathbf{w}_{1}, \mathbf{w}_{2}, \lambda_{1}, \lambda_{2}, \lambda_{3}\right)= \\
& r_{1} \lambda_{1}+r_{2} \lambda_{2}+r_{2} \lambda_{3}+\mathbf{w}_{1}^{H} \mathbf{A}_{1} \mathbf{w}_{1}+\mathbf{w}_{2}^{H} \mathbf{A}_{2} \mathbf{w}_{2},
\end{aligned}
$$

where $\lambda_{i}(i=1,2,3)$ are Lagrange multipliers, and

$$
\left\{\begin{array}{l}
\mathbf{A}_{1}=\mathbf{I}_{N}-\lambda_{1} \mathbf{h}_{1} \mathbf{h}_{1}^{H}+\lambda_{2} r_{2} \mathbf{h}_{1} \mathbf{h}_{1}^{H}+\lambda_{3} r_{2} \mathbf{h}_{2} \mathbf{h}_{2}^{H}, \\
\mathbf{A}_{2}=\mathbf{I}_{N}-\lambda_{2} \mathbf{h}_{1} \mathbf{h}_{1}^{H}-\lambda_{3} \mathbf{h}_{2} \mathbf{h}_{2}^{H} .
\end{array} .\right.
$$

Therefore, the dual problem of (4) can be written as

$$
\begin{array}{ll} 
& d^{*}=\max \quad r_{1} \lambda_{1}+r_{2} \lambda_{2}+r_{2} \lambda_{3} \\
\text { s.t. } & \mathbf{A}_{1} \succeq 0, \quad \mathbf{A}_{2} \succeq 0, \quad \lambda_{i} \geq 0, \quad i=1,2,3,
\end{array}
$$

and the Karush-Kuhn-Tucker (KKT) system is

$$
\left\{\begin{array}{l}
\mathbf{A}_{1} \mathbf{w}_{1}=\mathbf{0}, \mathbf{A}_{2} \mathbf{w}_{2}=\mathbf{0} \\
\text { Primary \& dual Constraints. } \\
\text { Complementary slackness }
\end{array}\right.
$$

Proposition 1. Given the optimal solution $\lambda_{1}^{*}, \lambda_{2}^{*}, \lambda_{3}^{*}$ of the dual optimization problem in (7), if $\mathbf{w}_{1}^{*}, \mathbf{w}_{2}^{*}$ satisfy the $K K T$ conditions in (8), then strong duality holds, i.e., $p^{*}=d^{*}$.

Proof: We first show that the optimization problem in (4) can be reformulated as a convex problem. The objective function and the first constraint are clearly convex functions

\footnotetext{
${ }^{1}$ The optimal decoding order can be found by comparing the required power by the cases with different order.
}

of the precoding vectors. To reveal the hidden convexity of the last two constraints, we follow the same steps developed in [14]. Note that the quadratic forms in the last two constraints make $\mathbf{w}_{i}$ and $e^{j \theta_{i}} \mathbf{w}_{i}$ equivalent for any phase rotation $\theta_{i}, i=$ 1,2 . By using this phase ambiguity, the constraints can be reformulated as second-order cone constraints. It is easy to show that Slater's constraint qualification is satisfied. Hence strong duality holds and $\mathbf{w}_{1}^{*}, \mathbf{w}_{2}^{*}$ are indeed global optimal.

The dual optimization problem in (7) is in a formulation of semi-definite programming (SDP), thus can be solved efficiently by interior point methods. Once the optimal solution of (7) is obtained, the KKT system in (8) can be solved efficiently via Newton's iterative method. By taking advantage of the strong duality, the resultant precoding vectors are global optimal.

\section{A. Dual Constraints Reduction}

\section{Closed-FORM Solutions}

In order to derive the closed-form solution of the optimization problem in (7), we first introduce a lemma and a corollary to reduce the semi-definite constraints.

Lemma 1. Assume $\mathbf{h}$ and $\mathbf{g}$ are two complex-valued vectors in $\mathbb{C}^{N}$. By defining $\cos ^{2} \theta=\frac{\mathbf{h}^{H} \mathbf{g g}^{H} \mathbf{h}}{\|\mathbf{h}\|^{2}\|\mathbf{g}\|^{2}}$, then $\mathbf{h} \mathbf{h}^{H}+\mathbf{g g}{ }^{H}$ has at most 2 non-zero eigenvalues:

$\frac{1}{2}\left(\|\mathbf{h}\|^{2}+\|\mathbf{g}\|^{2} \pm \sqrt{\left(\|\mathbf{h}\|^{2}+\|\mathbf{g}\|^{2}\right)^{2}-4\|\mathbf{h}\|^{2}\|\mathbf{g}\|^{2} \sin ^{2} \theta}\right)$.

Proof: It is obvious that $\mathbf{h} \mathbf{h}^{H}+\mathbf{g g}^{H}$ has at most 2 nonzero eigenvalues $\phi_{1}, \phi_{2}$. We have

$$
\phi_{1}+\phi_{2}=\operatorname{tr}\left(\mathbf{h h}^{H}+\mathbf{g g}^{H}\right)=\|\mathbf{h}\|^{2}+\|\mathbf{g}\|^{2}
$$

and

$\phi_{1}^{2}+\phi_{2}^{2}=\operatorname{tr}\left(\left(\mathbf{h} \mathbf{h}^{H}+\mathbf{g g}^{H}\right)^{2}\right)=\|\mathbf{h}\|^{4}+\|\mathbf{g}\|^{4}+2 \mathbf{h}^{H} \mathbf{g g}^{H} \mathbf{h}$.

Therefore, we have

$$
\phi_{1} \phi_{2}=\frac{1}{2}\left(\left(\phi_{1}+\phi_{2}\right)^{2}-\left(\phi_{1}^{2}+\phi_{2}^{2}\right)\right)=\|\mathbf{h}\|^{2}\|\mathbf{g}\|^{2} \sin ^{2} \theta \text {. }
$$

By combining (9) and (10), the lemma is proved. This lemma can also be found in [15].

By applying the methodology in the proof of Lemma 1, we have the following corollary for obtaining the eigenvalues of $\mathbf{h h}^{H}-\mathbf{g g}^{H}$.

Corollary 1. Assume $\mathbf{h}$ and $\mathbf{g}$ are two complex-valued vectors in $\mathbb{C}^{N}$. Then $\mathbf{h h}^{H}-\mathrm{gg}^{H}$ has at most 2 non-zero eigenvalues:

$\frac{1}{2}\left(\|\mathbf{h}\|^{2}+\|\mathbf{g}\|^{2} \pm \sqrt{\left(\|\mathbf{h}\|^{2}+\|\mathbf{g}\|^{2}\right)^{2}-4\|\mathbf{h}\|^{2}\|\mathbf{g}\|^{2}\left(1+\cos ^{2} \theta\right)}\right)$.

By employing Lemma 1 and Corollary 1, the dual constraints can be reduced as

$$
\left\{\begin{array}{l}
\left\|\mathbf{k}_{1}\right\|^{2}+\left\|\mathbf{k}_{2}\right\|^{2} \leq \min \left(1+\left\|\mathbf{k}_{1}\right\|^{2}\left\|\mathbf{k}_{2}\right\|^{2}\left(1+\cos ^{2} \theta\right), 2\right) \\
\left\|\mathbf{l}_{1}\right\|^{2}+\left\|\mathbf{l}_{2}\right\|^{2} \leq \min \left(1+\left\|\mathbf{l}_{1}\right\|^{2}\left\|\mathbf{l}_{2}\right\|^{2} \sin ^{2} \theta, 2\right) \\
\lambda_{i} \geq 0 \quad i=1,2,3
\end{array},\right.
$$

where

$$
\left\{\begin{array}{l}
\mathbf{k}_{1}=\sqrt{\lambda_{1}-\lambda_{2} r_{2}} \mathbf{h}, \quad \mathbf{k}_{2}=\sqrt{\lambda_{3} r_{2}} \mathbf{h}_{2} \\
\mathbf{l}_{1}=\sqrt{\lambda_{2}} \mathbf{h}_{1} \quad \mathbf{l}_{2}=\sqrt{\lambda_{3}} \mathbf{h}_{2} \\
\cos ^{2} \theta=\frac{\mathbf{h}_{1}^{H} \mathbf{h}_{2} \mathbf{h}_{2}^{H} \mathbf{h}_{1}}{\left\|\mathbf{h}_{1}\right\|^{2}\left\|\mathbf{h}_{2}\right\|^{2}}
\end{array} .\right.
$$




\section{B. Optimal Closed-form Solutions}

In this subsection, by applying the reduced semi-definite constraints, the global optimal closed-form solution of (7) will be derived for some special cases. Among these special cases, the definition of quasi-degraded channels will be introduced, and its physical importance as well as its properties will be discussed in detail.

The closed-form solutions of the dual QoS optimization problem in (7) in general is difficult to obtain. However, it will be shown that the optimal closed-form solution can be attained, by studying this optimization problem in (7) with 3 different solution subsets ${ }^{2}$, i.e., $\lambda_{2}=0, \lambda_{3}=0$, and $\lambda_{i} \neq 0, i=1,2,3$. Proposition 2 and Proposition 3 provide the optimal closedform solutions for the subsets 1 and 2, respectively.

Proposition 2. If $\lambda_{2}=0$, then the optimal solution is

$$
\lambda_{1}=\left\|\mathbf{h}_{1}\right\|^{-2} \frac{1+r_{2}}{1+r_{2} \sin ^{2} \theta}, \quad \lambda_{3}=\left\|\mathbf{h}_{2}\right\|^{-2} .
$$

The optimal value of the objective function is

$$
d^{*}=\frac{r_{2}}{\left\|\mathbf{h}_{2}\right\|^{2}}+\frac{r_{1}}{\left\|\mathbf{h}_{1}\right\|^{2}} \frac{1+r_{2}}{1+r_{2} \sin ^{2} \theta} .
$$

Proof: By using Lemma 1 and Corollary 1, the dual constraints can be reduced as

$$
\left\{\begin{array}{l}
\lambda_{3} \leq\left\|\mathbf{h}_{2}\right\|^{-2}, \quad \lambda_{i} \geq 0, \quad i=1,3 . \\
\lambda_{1} \leq \frac{1}{\left\|\mathbf{h}_{1}\right\|^{2}} \frac{1+\lambda_{3} r_{2}\left\|\mathbf{h}_{2}\right\|^{2}}{1+\lambda_{3} r_{2}\left\|\mathbf{h}_{2}\right\|^{2} \sin ^{2} \theta}
\end{array} .\right.
$$

Note that $\frac{1}{\left\|\mathbf{h}_{1}\right\|^{2}} \frac{1+\lambda_{3} r_{2}\left\|\mathbf{h}_{2}\right\|^{2}}{1+\lambda_{3} r_{2}\left\|\mathbf{h}_{2}\right\|^{2} \sin ^{2} \theta}$ is monotonically increasing with $\lambda_{3}$ since that $\left\|\mathbf{h}_{2}\right\|^{2} \geq \mathbf{h}_{2} \|^{2} \sin ^{2} \theta$ always holds. Therefore, $\lambda_{1}, \lambda_{3}$ can be simultaneously maximized and the proof is completed.

The case with $\lambda_{2}=0$ has an important meaning in practice, as explained in the following. To better illustrate the case of $\lambda_{2}=0$, we define a term, quasi-degraded channels, as introduced in the following:

Definition 1. Given the channel coefficients $\mathbf{h}_{1}, \mathbf{h}_{2}$ and the target interference levels $r_{1}, r_{2}$, the broadcast channels $\mathbf{h}_{1}$ and $\mathbf{h}_{2}$ with respect to $r_{1}$ and $r_{2}$ are quasi-degraded if the optimal solution of the dual QoS optimization problem in (7) satisfies $\lambda_{2}=0$.

By using this definition, we can have four remarks for Proposition 2 as follows:

Remark 1: the closed-form QoS solution of quasi-degraded broadcast channels can be obtained, as illustrated in Proposition 2.

Remark 2: for a heterogeneous ${ }^{3}$ broadcast system, the case with quasi-degraded channels appears with a considerably high probability, as verified by the computer simulations shown in Table I. Particularly, Table I illustrates the probability to have quasi-degraded channels versus $\sigma_{1}$. In this simulation, the target interference levels are set as $\left(r_{1}, r_{2}\right)=(1,1)$, and the variance of the channel coefficient of user 2 is fixed to be

\footnotetext{
${ }^{2}$ Note that $\lambda_{1} \neq 0$, and other cases are not needed.

${ }^{3}$ We say a broadcast system to be heterogeneous if the channel coefficients of different users are not identically distributed, i.e., their variances are different, $\sigma_{1}^{2} \neq \sigma_{2}^{2}$.
}

TABLE I: Quasi-degraded probability versus $\sigma_{1}$

\begin{tabular}{|c|c|c|c|c|c|c|}
\hline $\operatorname{Pr} \sigma_{1}$ & 3 & 4 & 5 & 6 & 7 & 8 \\
\hline $\mathrm{N}$ & & & & & & \\
\hline 2 & 0.6900 & 0.7979 & 0.8587 & 0.9006 & 0.9283 & 0.9451 \\
\hline 4 & 0.4513 & 0.6264 & 0.7389 & 0.8034 & 0.8488 & 0.8891 \\
\hline 6 & 0.3012 & 0.4913 & 0.634 & 0.7168 & 0.7901 & 0.8329 \\
\hline
\end{tabular}

unity, i.e., $\sigma_{2}^{2}=1$. One can observe that the more disperse the broadcast system is, the larger probability the case with quasi-degraded channels appears.

Remark 3: if the broadcast channels are quasi-degraded, the proposed optimization algorithm in combination with superposition coding and SIC can achieve the same performance as DPC, as explained in the following. Since $\lambda_{2}=0$, the optimization problem in (4) is equivalent to

$$
\begin{array}{ll} 
& \min \quad\left\|\mathbf{w}_{1}\right\|^{2}+\left\|\mathbf{w}_{2}\right\|^{2} \\
\text { s.t. } & \mathbf{h}_{1}^{H} \mathbf{w}_{1} \mathbf{w}_{1}^{H} \mathbf{h}_{1} \geq r_{1} \\
& \mathbf{h}_{2}^{H} \mathbf{w}_{2} \mathbf{w}_{2}^{H} \mathbf{h}_{2} \geq r_{2}+r_{2} \mathbf{h}_{2}^{H} \mathbf{w}_{1} \mathbf{w}_{1}^{H} \mathbf{h}_{2},
\end{array}
$$

which is exactly the same as the power minimization problem for DPC in MISO systems. Note that the minimal required power of quasi-degraded channels is the same as that of DPC, and the quasi-degraded channels appear with a considerably high probability in a heterogeneous broadcast system. Therefore, the overall performance achieved by the proposed scheme is only slightly outperformed by the optimal DPC. Insightfully, although MISO broadcast channels are not degraded, it is also possible to achieve the optimal performance only using NOMA instead of DPC, provided that the broadcast channels are quasi-degraded.

Remark 4: On one hand, when the channels are quasidegraded, the closed-form solution $\left(\lambda_{1}, \lambda_{2}, \lambda_{3}\right)$ of the dual problem in (7) can be obtained according to Proposition 2. By applying the closed-form solution, $\mathbf{A}_{1}, \mathbf{A}_{2}$ are obtained. By considering the KKT system in (8), firstly, one can observe that $\mathbf{w}_{1}$ and $\mathbf{w}_{2}$ are in the null space of $\mathbf{A}_{1}$ and $\mathbf{A}_{2}$, respectively. Then, by considering the complementary slackness, the optimal precoding vectors can be obtained efficiently by Newton's iterative method, which significantly reduces the computational complexity of the conventional interior method. On the other hand, when the channels are not quasi-degraded, since we have no closed-form solution, the conventional interior method is adopted. In conclusion, with regard to a heterogeneous broadcast system, the overall computational complexity is also significantly reduced since the quasi-degraded channels appear with a considerably high probability.

Proposition 3. If $\lambda_{3}=0$, then the optimal solution is

$$
\lambda_{1}=\left\|\mathbf{h}_{1}\right\|^{-2}\left(1+r_{2}\right), \quad \lambda_{2}=\left\|\mathbf{h}_{1}\right\|^{-2} .
$$

The optimal value of the objective function is

$$
d^{*}=\left\|\mathbf{h}_{1}\right\|^{-2}\left(r_{1}+r_{2}+r_{1} r_{2}\right) .
$$

Proof: By using Lemma 1 and Corollary 1, the dual constraints can be reduced as

$\left(\lambda_{1}-\lambda_{2} r_{2}\right)\left\|\mathbf{h}_{1}\right\|^{2} \leq 1, \quad \lambda_{2}\left\|\mathbf{h}_{1}\right\|^{2} \leq 1, \quad \lambda_{i} \geq 0, \quad i=1,2$. 
Obviously, it can be transformed as

$\lambda_{1} \leq\left\|\mathbf{h}_{1}\right\|^{-2}\left(1+r_{2}\right), \quad \lambda_{2} \leq\left\|\mathbf{h}_{1}\right\|^{-2}, \quad \lambda_{i} \geq 0, \quad i=1,2$.

Therefore, the optimal value of the objective function is $d^{*}=$ $\left\|\mathbf{h}_{1}\right\|^{-2}\left(r_{1}+r_{2}+r_{1} r_{2}\right)$, and the proof is completed.

Remark 5: It is important to point out that this case often implies that a wrong decoding order is used. Or in other words, if $\lambda_{3}=0$, then, it is more likely that we can obtain a smaller required power by changing the decoding order.

\section{Simulation Results}

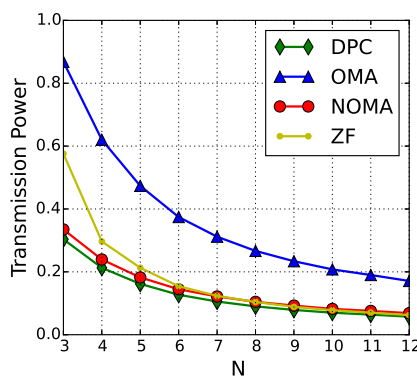

(a) $\left(r_{1}, r_{2}, \sigma_{1}, \sigma_{2}\right)=(1,1,2,1)$

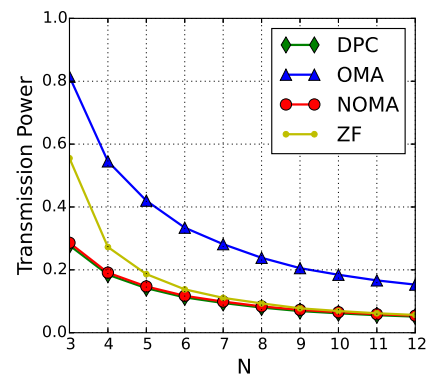

(b) $\left(r_{1}, r_{2}, \sigma_{1}, \sigma_{2}\right)=(1,1,3,1)$
Fig. 1: Power consumption for MISO broadcast systems

To measure the performance of the optimal NOMA precoding algorithm in terms of power efficiency, the total power consumption of different precoding algorithms for MISO broadcast systems versus $N$ is plotted in Fig. 1(a) and Fig. 1(b). It is observed that the optimal NOMA strategy has a significant improvement in terms of power consumption in comparison with the traditional OMA scheme (TDMA/FDMA). It is also noted that the optimal NOMA strategy only has a slight performance loss compared to the optimal DPC. In comparison with the zero-forcing algorithm ${ }^{4}$, the proposed NOMA strategy yields a significant performance gain when $N$ is small, and the two have similar performance when $N$ is large. It is also worthy pointing out their difference compared to DPC or ZFBF for large $N$ gets more negligible when $\frac{\sigma_{1}^{2}}{\sigma_{2}^{2}}$ becomes larger.

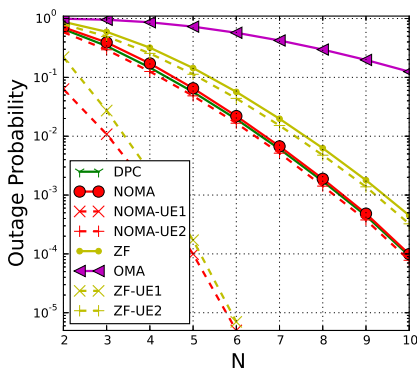

(a) Robustness

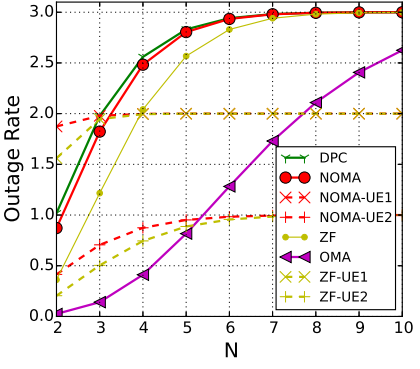

(b) Outage rate
Fig. 2: Performance comparison for MISO broadcast systems

To measure the robustness and outage rate performance of the proposed optimal NOMA algorithm, the outage probability with the fixed total power and outage rate versus $N$ is plotted in Fig. 2(a) and Fig. 2(b), respectively. Consider a 2-user MISO broadcast system, where the BS is located at the center, user 1 and user 2 are uniformly distributed in a circle and

${ }^{4} \mathrm{In} \mathrm{ZFBF}$, the optimal power allocation is adopted. a ring, respectively. Denote the distance between the BS and user $i$ by $d_{i}(i=1,2)$. The channel coefficients are modelled as $\sigma_{i}^{2}=\frac{5}{1+d_{i}^{3}}$, where $d_{1} \leq 1$ and $2 \leq d_{2} \leq 3$. Given the maximum transmit power $P_{\max }$ and a pair of target interference levels $r_{1}, r_{2}$, the outage probability is

$$
p_{\text {out }}\left(P_{\max }, r_{1}, r_{2}\right):=\mathbb{P}\left(\min \left\|\mathbf{w}_{1}\right\|^{2}+\left\|\mathbf{w}_{2}\right\|^{2}>P_{\max }\right) .
$$

Especially, in Fig. 2(b), the maximum transmit power is set to be 1 , i.e., $P_{\max }=1$, and the target interference levels are set as $\left(r_{1}, r_{2}\right)=(2,1)$. It is observed from these figures that the proposed NOMA strategy achieves a performance similar to DPC, and outperforms all the other precoding algorithms, such as zero-forcing beamforming and traditional OMA.

\section{CONCLUSION}

In this paper, a quality-of-service (QoS) optimization problem for 2-user MISO-NOMA systems was investigated. Given a pair of target interference levels, the minimum required power and the optimal precoding vectors have been generally obtained by solving the dual QoS optimization problem and via Newton's iterative algorithm. Additionally, the quasi-degraded definition was introduced, as well as its physical meaning and excellent properties. Finally, simulation results were provided to show that the proposed precoding algorithm can outperform many existing schemes, and yield a performance close to DPC.

\section{REFERENCES}

[1] Q. C. Li, H. Niu, A. T. Papathanassiou, and G. Wu, "5G network capacity: key elements and technologies," IEEE Veh. Technol. Mag., vol. 9, no. 1, pp. 71-78, 2014.

[2] 3rd Generation Partnership Project (3GPP), Study on downlink multiuser superposition transmission for LTE, Mar. 2015.

[3] Y. Saito, Y. Kishiyama, A. Benjebbour, T. Nakamura, A. Li, and K. Higuchi, "Non-orthogonal multiple access (NOMA) for cellular future radio access," in Proc. IEEE Vehicular Technology Conference (VTC Spring), Jun. 2013.

[4] Z. Ding, P. Fan, and V. Poor, "Impact of user pairing on $5 \mathrm{G}$ nonorthogonal multiple access downlink transmissions," IEEE Trans. Veh. Technol., vol. PP, no. 99, pp. 1-1, 2015.

[5] Z. Ding, Z. Yang, P. Fan, and H. V. Poor, "On the performance of non-orthogonal multiple access in $5 \mathrm{G}$ systems with randomly deployed users," IEEE Signal Process. Lett., vol. 21, no. 12, pp. 1501-1505, 2014.

[6] T. M. Cover and J. A. Thomas, Elements of information theory. John Wiley \& Sons, 2012.

[7] M. H. Costa, "Writing on dirty paper (corresp.)," IEEE Trans. Inf. Theory, vol. 29, no. 3, pp. 439-441, 1983.

[8] H. Weingarten, Y. Steinberg, and S. Shamai, "The capacity region of the Gaussian multiple-input multiple-output broadcast channel," IEEE Trans. Inf. Theory, vol. 52, no. 9, pp. 3936-3964, 2006.

[9] Q. Sun, S. Han, C.-L. I, and Z. Pan, "On the ergodic capacity of MIMO NOMA systems," IEEE Wireless Commun. Lett, vol. 4, no. 4, pp. 405408, Aug. 2015.

[10] M. F. Hanif, Z. Ding, T. Ratnarajah, and G. K. Karagiannidis, "A minorization-maximization method for optimizing sum rate in the downlink of non-orthogonal multiple access systems," IEEE Trans. Signal Process., vol. 64, no. 1, pp. 76-88, 2016.

[11] Y. Liu, Z. Ding, M. Elkashlan, and H. V. Poor, "Cooperative nonorthogonal multiple access with simultaneous wireless information and power transfer," IEEE J. Sel. Areas Commun., to appear in 2016.

[12] J. Choi, "Minimum power multicast beamforming with superposition coding for multiresolution broadcast and application to NOMA systems," IEEE Trans. Commun., vol. 63, no. 3, pp. 791-800, 2015.

[13] S. Boyd and L. Vandenberghe, Convex optimization. Cambridge university press, 2004.

[14] E. Bjornson, M. Bengtsson, and B. Ottersten, "Optimal multiuser transmit beamforming: A difficult problem with a simple solution structure [lecture notes]," IEEE Signal Process. Mag., vol. 31, no. 4, pp. 142-148, 2014.

[15] J. Hudson, Adaptive array principles. IET, 1981, vol. 11. 